\title{
Intense Pulsed Light Treatment of Persistent Facial Hypermelanosis Following Drug-Induced Toxic Epidermal Necrolysis
}

\author{
Philippe Paquet, MD, PhD, and GÉrald E. Piérard, MD, PhD \\ Department of Dermatopathology, University Hospital of Liège, Liège, Belgium
}

BACKGROUND. Cutaneous hyperpigmentation is one of the most cosmetically disturbing sequel of drug-induced toxic epidermal necrolysis. Intense pulsed light is a promising tool for treating some melanocytic lesions.

OBJECTIVE. The objective was to assess the effect of intense pulsed light in treating post-toxic epidermal necrolysis facial hypermelanosis.

METHODS. Two Caucasian men aged 35 and 50 years presented with long-standing (32 and 39 years) severe hypermelanosis of the face after sulfonamide-induced toxic epidermal necrolysis. They were treated by intense pulsed light. Cutoff filters of 550, 590 , and $615 \mathrm{~nm}$ were employed for five intense pulsed light sessions at 4-week intervals. The treatment was characterized by energy fluence of 25 to $32 \mathrm{~J} / \mathrm{cm}^{2}$, pulse width of 2.2 to 3.2 $\mathrm{ms}$, and double- to triple-pulse mode respecting a $30-\mathrm{ms}$ delay.
Before intense pulsed light treatment, and 2 months after the fifth intense pulsed light session, clinical photographs and skin biopsies were performed in combination with quantitative narrow-band remittance spectrophotometry of melanin pigmentation. Patients were clinically followed-up for 8 months after the end of the treatment.

RESULTS. In both patients, clinical, histologic, and spectrophotometric assessments showed an average of $80 \%$ decrease in the hypermelanosis. No clinical recurrence of the hypermelanosis developed during the 8-month follow-up after intense pulsed light treatment. No major persistent side effects were experienced, especially hypopigmentation.

CONCLUSION. Intense pulsed light appears to be effective and safe for treating post-toxic epidermal necrolysis hypermelanosis in Caucasian patients.

PHILIPPE PAQUET, MD, PHD, AND GÉRALD E. PIÉRARD, MD, PHD HAVE INDICATED NO SIGNIFICANT INTEREST WITH COMMERCIAL SUPPORTERS.

DRUG-INDUCED TOXIC epidermal necrolysis is a rare, potentially lethal disease characterized by sudden epidermal necrosis. The only recognized cause of the disease is an adverse reaction to drugs, especially sulfonamides, phenytoin, nonsteroidal anti-inflammatory drugs, and allopurinol. ${ }^{1}$ The toxic epidermal necrolysis-induced death rate reaches approximately $30 \% .^{1}$ Long-term sequelae in the survivors remain frequent. ${ }^{1}$ Pulmonary, myocardial, osseous and gastrointestinal complications have also been described. ${ }^{1}$ The most common long-term consequences, however, affect the eyes, skin, oral and genital mucosae, and nails. ${ }^{2-4}$ Cutaneous sequelae include keloidal scars, alopecia, speckled eruptive melanocytic nevi, and hyper- or hypomelanosis. ${ }^{3,4}$

We report two adult patients presenting with longstanding hypermelanosis of the face following toxic epidermal necrolysis that developed during childhood. The hyperpigmentation was treated successfully by

Address correspondence and reprint requests to: Philippe Paquet, MD, Department of Dermatopathology, CHU Sart Tilman, B-4000 Liège, Belgium, or e-mail: P. Paquet@chu.ulg.ac.be. intense pulsed light. To the best of our knowledge, these cases are the first descriptions of post-toxic epidermal necrolysis hyperpigmentation treated by intense pulsed light. We also briefly discuss the pathogenesis of post-toxic epidermal necrolysis hypermelanosis.

\section{Case Report}

Two phototype II Caucasian men, aged 35 and 50 years, suffered from toxic epidermal necrolysis at the age of 3 and 11 years, respectively. The clinical presentation was similar for the two patients, including epidermal sloughing over $50 \%$ of the body surface, and severe involvement of the face, eyes, and mucous membranes. Trimethoprim-sulfamethoxazole of the sulfonamide-class antibiotic was identified as the culprit drug in both cases. Cutaneous biopsies taken at the onset of the disease confirmed the diagnosis of toxic epidermal necrolysis. After the early recovery phase of the disease, facial skin showed a scar texture with a persistent diffuse and severe hypermelanosis, particularly prominent on the cheeks. By contrast, the 
other parts of the body surface fully recovered without any pigmentary change.

An intense pulsed light device (Multilight ${ }^{\mathrm{T}}$, ESC Medical Systems Ltd, Yokneam, Israel) was used to treat the hypermelanosis in a five-session procedure with respective intervals of 4 to 6 weeks. Cutoff filters of 550, 590, and $615 \mathrm{~nm}$ were used. To deal with the superficial pigmentation, treatments started using the $550-\mathrm{nm}$ cutoff filter, a single pulse mode at $25 \mathrm{~J} / \mathrm{cm}^{2}$ fluence, and a pulse width of $3.6 \mathrm{~ms}$. To tackle deeper pigmentation when previous attempts with the shorter cutoff filter failed to clear the melanoderma, the cutoff filter was switched to 590 or $615 \mathrm{~nm} \mathrm{~A}$ double- or triple-pulse mode combined with a pulse width of 3.2 or $2.2 \mathrm{~ms}$, a $30-\mathrm{ms}$ delay, and a fluence of 25 to 30 and 26 to $32 \mathrm{~J} / \mathrm{cm}^{2}$ were employed using 590- and 615 -filters, respectively. The two cheeks were entirely treated with a $2.8 \times 0.8-\mathrm{cm}$ spot size, representing 25 to 35 pulses by session. The intense pulsed light spots overlapped by about $10 \%$. Before treatment, skin cooling was obtained using cold packs for $1 \mathrm{~min}$. In addition, a 2-mm-thick coating with a chilled colorless gel was applied to the treatment area to protect the epidermis from thermal injury and to help deliver the light uniformly at the skin surface. No local anesthesia was used. Patients were advised to apply a corticosteroid and antiseptic combination cream (Fucicort, Leo) twice daily for 7 days. In the period covering 2 months before treatment, the whole treatment phase, and 2 months after treatment, the patients were advised to avoid sun exposure and they regularly applied a sun-protective (SPF 60) product.

The treatment response was assessed by comparing pre- and posttreatment clinical photographs, cutaneous biopsies, and data from remittance spectrophotometry. Posttreatment evaluations were performed 2 and 8 months after the fifth intense pulsed light session. This period covered the summer season. Pre- and posttreatment biopsy specimens were taken close to each other. Sections were stained with Fontana-Masson silver stain to reveal melanin. A narrow-band spectrophotometer (Mexameter, $\mathrm{C}+\mathrm{K}$ Electronic, Cologne, France) was used according to the EEMCO recommendations. ${ }^{5}$ The melanin index was measured on two different sites of the cheeks. Melanin index assessments were precisely performed each time on the same sites. To avoid any influence of skin color variation due to skin tanning, drug and alcohol intake, physical exercise, and environmental temperature, results were expressed as a differential melanin index $(\Delta \mathrm{M})$ between each of the melanin index values obtained at the target sites and another selected area of normal looking skin on the anterior part of the neck. $\mathrm{M}$ and $\Delta \mathrm{M}$ values were expressed in arbi- trary units. The posttreatment $\Delta \mathrm{M}$ value was also calculated as a percentage relative to the pretreatment value.

\section{Results}

Deep purpuric spots developed rapidly after each intense pulsed light session. In some areas, especially at the beginning of the treatment, the patients developed blisters and crusts that resolved within 1 week leaving some discrete and transient depressed scars. After 3 months, the scars had cleared without leaving permanent marks.

The clinical assessment of treatment efficacy was supported by comparing the photographs. Overall, the two patients showed a marked clinical improvement of melanization after the fifth intense pulsed light session (Figure 1). The treatment did not induce any obvious hyper- or hypopigmentation. Eight months after the last intense pulsed light session, hypermelanosis did not recur even following summer.

The spectrophotometer assessments showed a sharp decrease in $\Delta \mathrm{M}$ (range, $-17 \%$ to $-117 \%$; mean, $-84 \%$ ) at completion of the treatment in both patients (Table 1). In each case, the initial histologic examination revealed a discrete superficial perivascular lymphoid infiltrate. The melanin overload in the deepest layers of the epidermis was accompanied by few melanophages in the superficial dermis. The FontanaMasson stain was strongly positive. After intense pulsed light treatment, a marked decrease of the intraepidermal melanin load was evidenced, but the reduction in dermal melanophages was less obvious (Figure 2).
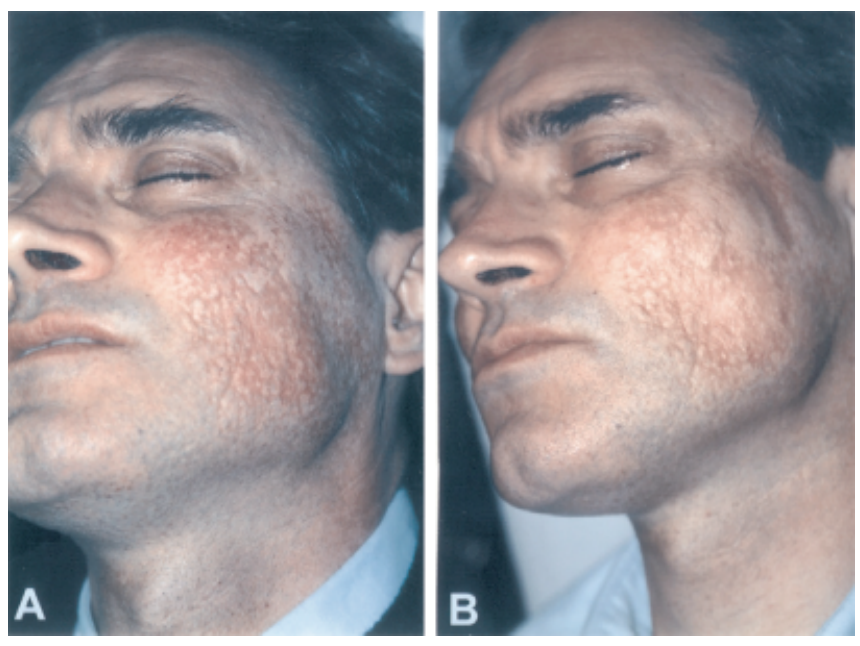

Figure 1. Post-toxic epidermal necrolysis facial hypermelanosis in Patient 2 before intense pulsed light treatment $(A)$ and after five intense pulsed light sessions (B). 
Table 1. Spectrophotometry Assessments of the Differential Melanin Index between the Hypermelanotic Cheeks and the Normal Looking Neck, Before and After Five Intense Pulsed Light Treatments

\begin{tabular}{|c|c|c|c|c|c|c|}
\hline & \multicolumn{3}{|c|}{ Left Cheek } & \multicolumn{3}{|c|}{ Right Cheek } \\
\hline & $\begin{array}{l}\text { Before Intense } \\
\text { Pulsed Light }\end{array}$ & $\begin{array}{l}\text { After Intense } \\
\text { Pulsed Light }\end{array}$ & Difference & $\begin{array}{l}\text { Before Intense } \\
\text { Pulsed Light }\end{array}$ & $\begin{array}{l}\text { After Intense } \\
\text { Pulsed Light }\end{array}$ & Difference \\
\hline \multicolumn{7}{|l|}{ Patient 1} \\
\hline External cheek & 658 & 325 & $333(-51 \%)$ & 815 & 174 & $641(-79 \%)$ \\
\hline Internal cheek & 506 & 422 & $84(-17 \%)$ & 1004 & -169 & $1173(-117 \%)$ \\
\hline \multicolumn{7}{|l|}{ Patient 2} \\
\hline External cheek & 715 & -6 & $721(-101 \%)$ & 956 & -46 & $1002(-104 \%)$ \\
\hline Internal cheek & 1043 & -146 & $1189(-114 \%)$ & 793 & 90 & $703(-89 \%)$ \\
\hline
\end{tabular}

\section{Discussion}

Changes in skin pigmentation including hypo- and hypermelanosis are among the most common disturbing cutaneous sequelae of toxic epidermal necrolysis. $^{3,4}$ The modifications in the melanocyte function are mainly seen on sun-exposed skin, although actinic exposure does not seem mandatory for their development. ${ }^{4}$

The precise pathogenesis of post-toxic epidermal necrolysis hyperpigmentation is still obscure. In many inflammatory diseases, epidermal melanocytes increase in number, size, dendricity, and melanin production. Arachidonic acid metabolites (prostaglandin E2, thromboxane B2, leukotrienes C and D4) and histamine, which are increased in amount in inflammatory conditions, are thought to play a key role in the induction of postinflammatory hypermelanosis. ${ }^{6,7}$ The vasoconstriction peptide endothelin-1 is also a strong keratinocyte-derived mitogen and melanogen for hu-

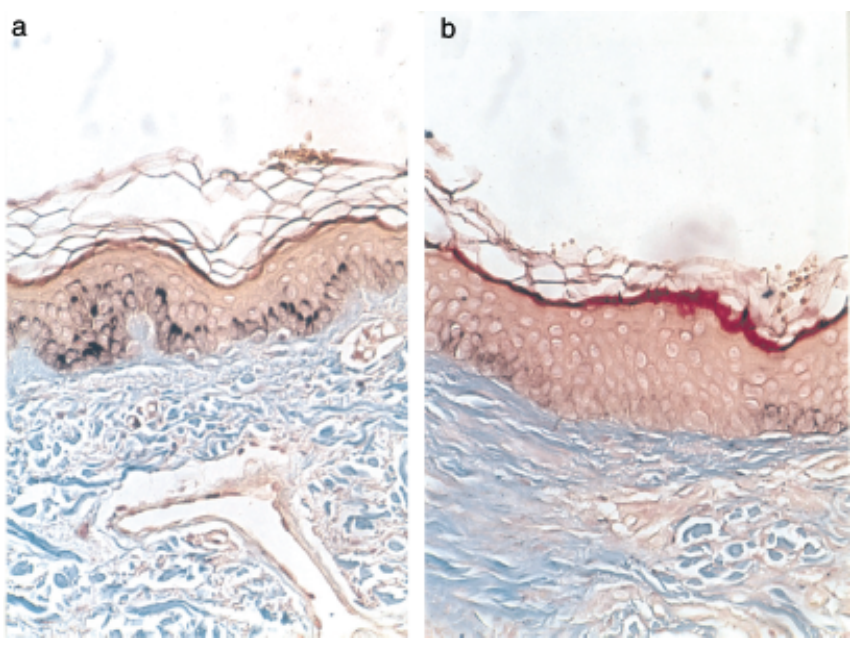

Figure 2. Fontana-Masson stain of cutaneous biopsies in Patient 2 before (a) and after five intense pulsed light sessions (b). Melanin deposits appear in black. man melanocytes. ${ }^{8}$ Endothelin-1 secretion is stimulated by tumor necrosis factor- $\alpha$, which is one of the main cytokines involved in toxic epidermal necrolysis pathogenesis. ${ }^{8}$ Nitric oxide might also be involved in the process. ${ }^{9}$ Indeed, any nitric oxide excess induces keratinocyte apoptosis and boosts melanocyte activity.

A direct effect of drug metabolites on epidermal melanocytes may also be involved in the increased melanin production. Indeed, contact between unirradiated melanocytes in culture and DNA-damaging agents or thymine dinucleotides, the by-products of DNA excision repair, is sufficient to increase the melanin content and up regulate tyrosinase gene expression. ${ }^{10}$ In addition, tyrosinase expression may be up regulated by removal of a repressor gene given the tyrosinase is under negative control in melanocytes. ${ }^{10}$

In our patients, the long-standing hypermelanosis resulted from toxic epidermal necrolysis developed during childhood and was exclusively located on the face. It should be noted that in children aged between 1 and 15 years, the number of active melanocytes is higher than in older subjects. The number of melanocytes also varies according to the region of the body, the cheeks exhibiting one of the highest densities. ${ }^{1}$ The persistence of hypermelanosis on sun-exposed skin in our patients suggests a ultraviolet-sustained pathomechanism. Moreover, the sulfonamides which are thought to be responsible for the presently reported toxic epidermal necrolysis cases are photosensitizing drugs. ${ }^{12}$ The mechanisms involved in ultravioletinduced hypermelanosis include melanocyte DNA damage and/or repair, keratinocyte-derived hyperproduction of the melanogenic factor endothelin-1, or the destruction of tyrosinase gene repressor protein PRP (photolyase regulatory protein). ${ }^{10}$

Dermal postinflammatory hypermelanosis results from melanin incontinence from the epidermis. Melanosomes are captured by dermal macrophages and dendrocytes. ${ }^{12}$ Among them, Factor XIIIa+ dendrocytes, whose population is particularly abundant in 
toxic epidermal necrolysis skin, have been shown to be able to store the released melanin products. ${ }^{13}$

Different kinds of lasers (Q-switched ruby, alexandrite, or Nd-Yag $)^{14-19}$ and intense pulsed light ${ }^{20-26}$ have been reported to be effective in treating some pigmented lesions, especially postinflammatory hyperpigmentation. There is, however, no information about the efficacy of intense pulsed light in treating posttoxic epidermal necrolysis hypermelanosis. The mechanism of action of these methods relies on the selective photothermolysis of pigmented cells. In contrast to lasers, intense pulsed light system offers the advantage of adjusting pulse width and wavelength according to the skin type and depth of pigment deposits in the skin. $^{20-26}$ In our patients, the melanin overload was mostly located in the epidermis. Thus, short wavelengths (cutoff filter of 550 and $590 \mathrm{~nm}$ ) were used preferentially. Clinical, histologic, and spectrophotometric assessments showed a prominent bleaching effect after five intense pulsed light sessions. This looks similar to the results obtained in treating postburn hyperpigmentation by intense pulsed light. ${ }^{26}$ There were no significant side effects, especially hypopigmentation. The long-term beneficial response of the treated area after sun exposure is difficult to predict because a reactivation of excessive melanization cannot be ruled out. Thus, the patients were advised to use lifetime potent sun-protective products during sunny days.

In conclusion, toxic epidermal necrolysis can be followed by persistent postinflammatory hypermelanosis especially on sun-exposed skin. Intense pulsed light appears to be a promising treatment for post-toxic epidermal necrolysis epidermal hyperpigmentation, with the possibility to precisely adjust the beam depth penetration according to the site of melanin deposits in the skin.

\section{References}

1. Fritsch O, Sidoroff A. Drug-induced Stevens-Johnson syndrome/ toxic epidermal necrolysis. Am J Clin Dermatol 2000;1:349-60.

2. Meneux E, Wolkenstein P, Haddad B, et al. Vulvovaginal involvement in toxic epidermal necrolysis: a retrospective study of 40 cases. Obstet Gynecol 1998;91:283-7.

3. Sheridan RL, Schulz JT, Ryan CM, et al. Long-term consequences of toxic epidermal necrolysis in children. Pediatrics 2002;109:74-8.

4. Paquet P, Arrese JE, Greimers R, Piérard GE. Eruptive speckled melanocytic naevi following drug-induced toxic epidermal necrolysis. Eur J Dermatol 1995;5:379-82.
5. Piérard GE. EEMCO guidance for the assessment of skin colour. J Eur Acad Dermatol Vener 1998;10:1-11.

6. Nordlund JJ. Postinflammatory hyperpigmentation. Dermatol Clin 1988;6:185-92.

7. Tomita Y, Maeda K, Tagami H. Mechanisms for hyperpigmentation in post-inflammatory pigmentation, urticaria pigmentosa and sunburn. Dermatologica 1989;179:S49-S53.

8. Manaka I, Kadono S, Kawashima M, et al. The mechanism of hyperpigmentation in seborrhoeic keratosis involves the high expression of endothelin-converting enzyme $1 \alpha$ and TNF- $\alpha$, which stimulate secretion of endothelin 1. Br J Dermatol 2001;145: 895-903.

9. Lerner L, Quresh AA, Reddy BV, Lerner EA. Nitric oxide synthase in toxic epidermal necrolysis and Stevens-Johnson syndrome. J Invest Dermatol 2000;114:196-9.

10. Ferguson CA, Kidson SH. The regulation of tyrosinase gene transcription. Pigment Cell Res 1997;10:127-38.

11. Tyack ZF, Pegg S, Ziviani J. Postburn dyspigmentation: its assessment, management, and relationship to scarring: a review of the literature. J Burn Care Rehabil 1997;18:435-40.

12. Epstein JH. Postinflammatory hyperpigmentation. Clin Dermatol 1989;7:55-65.

13. Piérard GE, Arrese Estrada J, Piérard-Franchimont C, DeleixheMauhin F. Is there a link between dendrocytes, fibrosis and sclerosis? Dermatologica 1990;181:264-5.

14. Kopera D, Hohenleutner U. Ruby laser treatment of melasma and postinflammatory hyperpigmentation. Dermatol Surg 1995;21:994.

15. Goldman MP. Postsclerotherapy hyperpigmentation: treatment with a flashlamp-excited pulsed dye laser. J Dermatol Surg Oncol 1992;18:417-22.

16. Dierickx C, Goldman MP, Fitzpatrick RE. Laser treatment of erythematous/hypertrophic and pigmented scars in 26 patients. Plast Reconstr Surg 1995;95:84-90.

17. Tafazzoli A, Rostan EF, Goldman MP. Q-switched ruby laser treatment for postsclerotherapy hyperpigmentation. Dermatol Surg 2000;26:653-6.

18. Raulin C, Hellwig S, Schonermark MP. Hyperpigmented burn injuries of the face: treatment with the Q-switched ruby laser. Plast Reconstr Surg 1997;99:265-6.

19. Taylor CR, Anderson RR. Ineffective treatment of refractory melasma and postinflammatory hyperpigmentation by Q-switched ruby laser. J Dermatol Surg Oncol 1994;20:592-7.

20. Gold MH, Foster TD, Bell MW. Nevus spilus successfully treated with intense pulsed light source. Dermatol Surg 1999;25:254-5.

21. Bjerring P, Christiansen K. Intense pulsed light source for treatment of small melanocytic nevi and solar lentigines. J Cutan Laser Ther 2000;2:177-81.

22. Moreno-Arias GA, Ferrando J. Intense pulsed light for melanocytic lesions. Dermatol Surg 2001;27:397-400.

23. Negishi K, Wakamatsu S, Kushikata N, et al. Full-face photorejuvenation of photodamaged skin by intense pulsed light with integrated contact cooling: initial experiences in Asian patients. Lasers Surg Med 2002;30:298-305.

24. Kawada A, Shiraishi H, Asai M, et al. Clinical improvement of solar lentigines and ephelides with an intense pulsed light source. Dermatol Surg 2002;28:504-8.

25. Kawada A, Asai M, Kameyama H, et al. Videomicroscopic and histopathological investigation of intense pulsed light therapy for solar lentigines. J Dermatol Sci 2002;29:91-6.

26. Ho WS, Chan H, Ying SY, et al. Prospective study on treatment of postburn hyperpigmentation by intense pulsed light. Lasers Surg Med 2003;32:42-5. 